\title{
Heterosis and Inbreeding Depression for Seed Yield and its Related Morphological Traits in Linseed (Linum usitatisimum L.)
}

\author{
P.C. Yadav*, R.K. Yadav, Vishwanath, Yogesh Pandey and Sanjeev Kumar \\ Department of genetics and plant breeding, Chandra Shekhar Azad University of Agriculture \\ and Technology, Kanpur-208002, (U.P.), India \\ *Corresponding author
}

\begin{abstract}
A B S T R A C T
\end{abstract}
Generation mean analysis with six generations, a set of 60 treatments $\left(10 \mathrm{P}_{1}, 10 \mathrm{P}_{2}, 10 \mathrm{~F}_{1}\right.$, $10 \mathrm{~F}_{2}, 10 \mathrm{BC}_{1}$ and $10 \mathrm{BC}_{2}$ ) was chosen to study the nature and magnitude of gene effects for seed yield and yield attributing traits. The studies were undertaken on Randomized

\section{Keywords}

Heterosis,

Inbreeding

depression, Seed

Article Info

Accepted:

26 December 2017

Available Online:

10 January 2018
Block Design with three replications in Rabi 2015-16. The range of heterosis over better parent was observed from 9.07 to 50.57 per cent. Three crosses namely, NPHY 29 X LCK 87312, NP121 X RR 76 and NPRR271 X NO 356 showed significant positive heterosis. The heterosis over mid parent varied from 7.77 to 50.42 per cent. Out of 10 crosses three cross NPHY 29 X LCK 87312, NP121 X RR 76 and NPRR271 X NO 356 showed significant positive heterosis. The range of inbreeding depression varied from 14.78 to 53.37 per cent. Three crosses, NPHY 29 X LCK 87312, NP121 X RR 76 and NPRR 271 X NO 356 showed significant increase. For oil content (\%) The range of heterosis over better parent was observed from -12.91 to 11.18 percent. Out of 10 crosses, only one cross NPRR 402 X NP 8 showed significant positive heterosis. The range of inbreeding depression varied from -0.92 to -9.10 per cent. Three crosses, NP121 X RR 76, RL-28-1 X L-53 and AHUDEARA $170 \mathrm{X}$ SJKO 05. Showed significant decrease while three combinations namely, NPHY 29 X LCK 87312, NPRR 271 X NO 356 and NPRR 402 X NP 8 showed significant increase. Native estimates of heterotic effects may be observed in some traits may be attributed to inter-allelic interactions.

\section{Introduction}

Linseed (Linum usitatissimum L.) is a diploid $(2 \mathrm{n}=30$, genome size $\sim 370 \mathrm{Mb})$ selfpollinated annual oilseed plant. Linseed (Linum usitatissimum L.) is an important oilseed crop grown for both seed as well as fibre. It is mainly grown for oil purpose; it is traditionally grown for edible and industrial purposes in South East Asia (Turkmenistan, Afghanistan and India), Asia Minor and South
Russia. Recent medical researchers have found linseed as best herbal source of carbohydrates, Omega -3 and Omega- 6 fatty acids, which have beneficial effect on human metabolism. The seed of linseed is a very rich source of nutrients, which contain oil $(40 \%)$, protein $(26 \%)$, fibre $(14 \%)$, mucilage $(12 \%)$, water $(9 \%)$, minerals $(4 \%)$, potassium $(0.74 \%)$, phosphorous $(0.70 \%)$, magnesium $(0.38 \%)$, calcium $(0.21 \%)$, sulphur $(0.21 \%)$, along with sodium, chlorine, iron, zinc, copper 
etc in traces. Lignan (1.90-6.16 mg/g) is also found in the seed. The oil contains different fatty acids like alpha linolenic acid (omega-3) $53.21 \%$, linoleic acid (omega-6) $17 \%$, oleic acid $18.51 \%$, stearic acid $4.42 \%$ and palmitic acid $4-6 \%$. Linseed is the richest source of omega-3 fatty acid and it contains almost twice as much as of omega- 3 in fish oil. The ratio of omega- 3 and omega- 6 present in linseed is about $4: 1$, so this is a best herbal source of omega-3 for improvement in human metabolism. World over, linseed is an important crop grown over 27.29 lakh ha with production of 25.20 lakh tons and average productivity of $923 \mathrm{~kg} / \mathrm{ha}$, while national production of 1.525 lakh tons is from 3.38 lakh area ha with low productivity of 473 $\mathrm{kg} / \mathrm{ha}$ (Anonymous, 2015). As far as productivity is concerned, Indian average yield $(473 \mathrm{~kg} / \mathrm{ha})$ is far below the productivity of UK (1500 kg/ha), Germany (1429 kg/ha), Canada (1538 kg/ha), USA (1076 kg/ha) and China (1000 kg/ha). India is an important linseed producer, which contributes about $11.82 \%$ to world acreage producing about 7 $\%$ of world production.

\section{Materials and Methods}

\section{Generation mean analysis}

\section{Building up of material}

20 parents (10 pure line female and 10 pure line male) will be crossed to develop the material. To be evaluated each female and male will be crossed only once during Rabi season 2013-14 to produce seeds of 10 straight crosses.

Raising of $F_{1 s}$ material and development of back crosses

The seeds of 10 crosses will be sown to raise $F_{1}$ population in Rabi- 2014-15. Each $F_{1}$ will be back crossed with their respective parents to produce the seeds of $\mathrm{BC}_{1} \& \mathrm{BC}_{2}$. The parents will be maintained and fresh crosses will also be made during this season to procure $F_{1}$ seeds of straight crosses.

\section{Plan of the lay-out for experiment}

All the $10 \mathrm{~F}_{1}$ 's, $10 \mathrm{~F}_{2 \mathrm{~s}}, 10 \mathrm{BC}_{1 \mathrm{~s}} \& 10 \mathrm{BC}_{2 \mathrm{~s}}$ along with their 10 pure line female \& 10 pure line male parents, will be grown in randomized block design with three replication during Rabi 2015-16 at Nawabganj Farm of CSAUA\&T Kanpur. Such observation was reported by Hayman (1958) Jinks and Jones (1958).

Oil content was determined by soxhlet method A.O.A.C 1975 (2). There were analyzed separately for their oil content and fatty acid composition.

The data were reported mean of three replications. Methyl esters were prepared by the method of Luddy et al., (3).

Heterosis expressed as per-cent increase or decrease of $F_{1}$ hybrid over the best commercial check (standard heterosis) were computed as per the method of Turner, (1953) and Hayes et al., (1955).

Heterosis over check

$$
\overline{\mathrm{F}_{1}}-\overline{\mathrm{CC}}
$$

(Standard heterosis $)=$

\section{$\overline{\mathrm{CC}}$} x 100

Where: $\overline{\mathrm{F}_{1}}=$ mean performance of $\mathrm{F}_{1}, \overline{\mathrm{CC}}=$ mean performance of the best commercial check

The differences in the magnitude of heterosis were tested by following procedure given by Panse and Sukhatme (1967)

Critical difference for commercial check $=$ $(2 \mathrm{Me} / \mathrm{r})^{1 / 2} \times \mathrm{t}$ 
Where: $\mathrm{r}=$ Number of replications, $\mathrm{Me}=$ Error mean sum of square from analysis of variance table, $\mathrm{t}=$ Table $\mathrm{t}$ value at error degrees of freedom corresponding to $5 \%$ or $1 \%$ level of significance.

Inbreeding depression is estimated when both $F_{1}$ and $F_{2}$ generations of the same cross are available.

Inbreeding depression was measured as described by Miller and Marani (1963).

Inbreeding depression $=\frac{\overline{\mathrm{F}_{1}}-\overline{\mathrm{F}_{2}}}{\overline{\mathrm{F}_{1}}} \times 100$

Where: $F_{1}$ and $F_{2}$ are the mean values of $F_{1}$ and $F_{2}$ progeny respectively of the same cross for a given character. Inbreeding depression may be high, medium, low and nil depending on cross.

\section{Results and Discussion}

\section{Heterosis and inbreeding depression}

The estimates of heterosis over better parent and mid parent in $F_{1}$ generation was recorded in percentage and presented in Table 1.

For days to $50 \%$ flowering, plant height $(\mathrm{cm}$.) and days to maturity negative and significant values and for other traits the positive and significant values of heterosis were considered desirable. The characters wise results are summarized below-

\section{Days to $50 \%$ flowering}

Heterosis was worked out in per cent over better parent (earlier flowering parent). The extent of heterosis over better parent ranged from -6.80 to 6.63 percent. Out of 10 crosses, only one cross (NP 121 X RR 76) showed highly significant negative heterosis for early flowering. One cross (AHUDERA $170 \mathrm{X}$ SJKO 05) showed significant positive heterosis for late flowering.

The heterosis over mid parent ranged from 5.28 to 3.47 per cent. One cross (NP $121 \mathrm{X}$ RR 76) showed significant negative heterosis. The maximum negative heterosis was observed in cross (NP $121 \mathrm{X}$ RR 76). On the other hand, one cross (AHUDERA $170 \mathrm{X}$ SJKO 05) exhibited significant heterosis over mid parent for late flowering.

The range of inbreeding depression varied from -2.53 to 9.50 per cent. Five crosses showed inbreeding depression with respect to early flowering. Crosses NPRR $271 \mathrm{X}$ NO 356, NPRR 402 X NP 8, RL-28-1 X L-53, NPHY $39 X$ NO 3 and NPHY 38 X LMHS-5 55 revealed significant positive values over $F_{1}$ early flowering whereas crosses NPHY $29 \mathrm{X}$ LCK 87312, NP121 X RR 76 and POLF $10 X$ SJKO 55 exhibited highest negative values of inbreeding depression for late flowering. Such observations were reported by Saraswat, A.V and Kumar, S. (1993), Patil and Chopde (1982) and Yadav e. al. (2000).

\section{Plant height (cm)}

The heterosis over better parent varied from 15.66 to 45.02 per cent. Out of 10 crosses, only one cross (NPHY 29 X LCK 87312) showed highly significant negative heterosis for dwarf plant stature. On the other hand, three crosses (NP 121 X RR 76, NPRR 402 X NP 8 and RL-28-1 X L-53) showed positive significant heterosis for tall plant stature.

The heterosis over mid parent varied from 14.37 to 45.39 per cent. The heterosis for over mid parent none of the cross showed highly significant negative heterosis for dwarf plant stature. Crosses (NP 121 X RR 76, NPRR 402 X NP 8 and RL-28-1 X L-53) showed positive significant heterosis for tall plant stature. 
The range of inbreeding depression varied from 2.01 to 49.63 per cent. Nine crosses NPHY 29 X LCK 87312, NP121 X RR 76, NPRR 271 X NO 356, NPRR 402 X NP 8, RL-28-1 X L-53, AHUDEARA 170 X SJKO 05, NPHY $39 \quad X \quad N O 3$, NPHY $28 \quad X$ 141NO18XRR9 and NPHY 38 LMHS-5 showed significant decrease while one combination namely, POLF 10 X SJKO 55 showed significant increase.

\section{Days to maturity}

The heterosis ranged from -8.33 to 6.29 and 5.46 to 8.72 per cent over both better and mid parent, respectively.

Out of 10 crosses, four crosses over better parent and three crosses over mid parent showed significant and negative heterosis for early maturity.

Crosses NPHY 29 X LCK 87312, NP 121 X RR 76, NP271 X NO 356 and AHUDERA 170 X SJKO 05 showed early maturity over better parent whereas crosses NPHY 29 X LCK 87312, NP 121 X RR 76 and NPHY 38 $\mathrm{X}$ LMHS-5 showed early duration over mid parent.

The range of inbreeding depression varied from 0.00 to -16.57 per cent. Eight crosses NPHY 29 X LCK 87312, NP121 X RR 76, NPRR 271 X NO 356, NPRR 402 X NP 8, RL-28-1 X L-53, AHUDEARA 170 X SJKO 05, NPHY 28 X 141NO18XRR9 and NPHY 38 LMHS-5 showed significant increase. Such observations were reported by Verma and Sinha (1993) and Shull (1908).

\section{Number of primary branches per plant}

The range of heterosis over better parent was observed from -9.09 to 170.00 per cent. Only four crosses AHUDERA 170 X SJKO 05, NPHY 39 X NO 3, NPHY 38 X LMHS-5 and
POLF 10 X SJKO 55 showed singificant positive heterosis.

The heterosis over mid parent varied from 40.74 to 134.78 per cent. Crosses NPHY $39 \mathrm{X}$ NO 3 and NPHY 38 X LMHS-5 showed significant positive heterosis.

The range of inbreeding depression varied from 0.00 to 59.25 per cent. Two crosses NPHY 29 X LCK 87312 and NPRR 402 X NP 8 showed significant decrease while seven combinations namely, NP121 X RR 76, RL28-1 X L-53, AHUDEARA 170 X SJKO 05, NPHY $39 \quad X \quad N O \quad 3$, NPHY $28 \quad X$ 141NO18XRR9, NPHY 38 LMHS-5 and POLF $10 \mathrm{X}$ SJKO 55 showed significant increase.

\section{Number of secondary branches per plant:}

The range of heterosis over better parent was observed from -46.48 to 342.85 per cent. Only five crosses out of 10 viz., NP 121 X RR 76, RL-28-1 X L-53, AHUDERA 170 X SJKO 05, NPHY 28 X 141NO18XRR9 and POLF $10 \mathrm{X}$ SJKO 55 showed significant positive heterosis.

The heterosis over mid parent varied from 41.74 to 239.72 per cent. Crosses NPHY 29 X LCK 87312, RL-28-1 X L-53, AHUDERA 170 X SJKO 05, NPHY 39 X NO 3, NPHY 28 $X$ 141NO18XRR9, NPHYB 38 X LMHS-5 and POLF $10 \mathrm{X}$ SJKO 55 showed significant positive heterosis.

The range of inbreeding depression varied from 10.00 to 60.87 per cent. All the crosses NPHY 29 X LCK 87312, NP121 X RR 76, NPRR 271 X NO 356, NPRR 402 X NP 8, RL-28-1 X L-53, AHUDEARA 170 X SJKO 05, NPHY $39 \quad X \quad N O 3$, NPHY $28 \quad X$ 141NO18XRR9, NPHY 38 LMHS-5 and POLF $10 \mathrm{X}$ SJKO 55 showed significant increase. 


\section{Number of capsules per plant}

The range of heterosis over better parent was observed from -37.40 to 172.10 per cent. Only four crosses RL-28-1 X L-53, AHUDERA $170 \mathrm{X}$ SJKO 05, NPHYB $38 \mathrm{X}$ LMHS-5 and POLF $10 \mathrm{X}$ SJKO 55 showed significant positive heterosis.

The heterosis over mid parent varied from 31.30 to 148.55 per cent. Crosses RL-28-1 X L-53, AHUDERA 170 X SJKO 05, NPHYB $38 \mathrm{X}$ LMHS-5 and POLF $10 \mathrm{X}$ SJKO 55 showed significant positive heterosis.

The range of inbreeding depression varied from 19.74 to 69.05 per cent crosses NPHY 29 X LCK 87312, NP121 X RR 76, NPRR 271 X NO 356, NPRR 402 X NP 8, RL-28-1 X L-53, AHUDEARA 170 X SJKO 05, NPHY 39 X NO 3, NPHY 28 X 141NO18XRR9, NPHY 38 LMHS-5 and POLF 10 X SJKO 55 showed significant increase.

\section{Number of seeds per capsule}

The heterosis over better parent varied from 25.92 to 14.28 per cent. Out of 10 crosses, none of the cross showed significant positive heterosis.

The heterosis over mid parent varied from 18.36 to 6.66 per cent. Out of 10 crosses, only one cross NPHY 39 X NO 3 showed significant negative heterosis.

The range of inbreeding depression varied from 19.74 to 69.05 per cent. Four crosses NPHY 29 X LCK 87312, NP121 X RR 76, NPHY $39 X$ NO 3 and POLF 10 X SJKO 55 showed significant decrease while six combinations namely, NPRR 271 X NO 356, NPRR 402 X NP 8, RL-28-1 X L-53, AHUDEARA 170 X SJKO 05, NPHY 28 X 141NO18XRR9 and NPHY 38 LMHS-5 showed significant increase.

\section{0-seed weight (g)}

The range of heterosis over better parent was observed from -10.55 to 6.49 per cent.

Out of 10 crosses, none of the parent showed significant positive heterosis for number of seeds per capsule.

The heterosis over mid parent varied from 11.22 to 3.53 percent Out of 10 crosses, none of the cross showed significant positive heterosis.

The range of inbreeding depression varied from -0.10 to 9.78 per cent. Five crosses NPRR 402 X NP 8, AHUDEARA $170 \mathrm{X}$ SJKO 05, NPHY 28 X 141NO18XRR9, NPHY 38 LMHS-5 and POLF 10 X SJKO 55 showed significant decrease while five combinations namely, NPHY 29 X LCK 87312, NP121 X RR 76, NPRR 271 X NO 356, RL-28-1 X L-53 and NPHY 39 X NO 3 showed significant increase.

\section{Seed yield per plant (g)}

The range of heterosis over better parent was observed from 9.07 to 50.57 per cent.

Eight crosses namely, NPHY 29 X LCK 87312, NP121 X RR 76, NPRR271 X NO 356, RL-28-1 X L-53, AHUDER 170 X SJKO 05, NPHY 39 X NO 3, NPHYNPHY $28 X$ 141NO18XRR9 and POLF $10 \times$ SJKO 55 showed significant positive heterosis.

The heterosis over mid parent varied from 7.77 to 50.42 per cent. Out of 10 crosses, NPHY 29 X LCK 87312, NP121 X RR 76, NPRR271 X NO 356, RL-28-1 X L-53, AHUDERA 170 X SJKO 05, NPHY 39 X NO 3 , NPHY 28 X 141NO18XRR9 and NPHYB 38 X LMHS-5 showed significant positive heterosis. The range of inbreeding depression varied from 14.78 to 53.37 per cent. 
Table.1 Estimates of heterosis over better parents, mid parent and inbreeding depression in percent for 16 characters in linseed

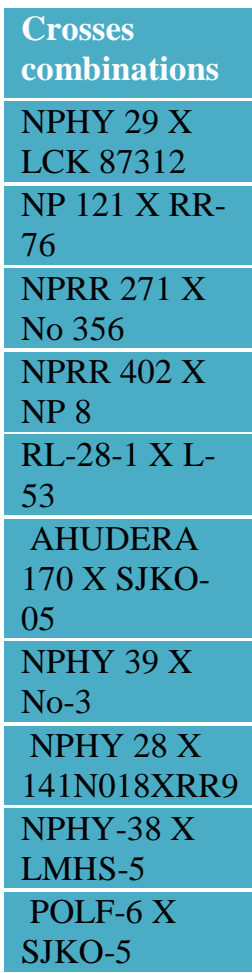

\begin{tabular}{|c|c|c|c|c|c|c|c|c|}
\hline \multicolumn{3}{|c|}{ Days to $50 \%$ flowering } & \multicolumn{3}{|c|}{ Plant height (cm) } & \multicolumn{3}{|c|}{ Days to maturity } \\
\hline BP & MP & ID & BP & MP & ID & BP & MP & ID \\
\hline-0.41 & 0.00 & 8.64 & $-15.66^{*}$ & -14.37 & 18.09 & $-8.33^{* *}$ & $-5.46^{* *}$ & -7.43 \\
\hline$-6.80^{* *}$ & $-5.28 *$ & 3.43 & $45.02^{* *}$ & $19.47 *$ & 49.63 & $-3.84 *$ & $-3.10^{*}$ & -5.60 \\
\hline-0.82 & 2.77 & -3.31 & 10.45 & -0.47 & 21.59 & $-3.70 *$ & 0.00 & -5.38 \\
\hline 0.00 & 0.00 & -2.53 & $42.93 * *$ & $25.96^{* *}$ & 38.71 & -1.52 & 0.38 & -6.97 \\
\hline 1.24 & 1.66 & -4.08 & $39.90^{* *}$ & $45.39^{* *}$ & 33.03 & 1.78 & 0.88 & -8.02 \\
\hline $6.63 * *$ & $5.01 *$ & 5.39 & -7.02 & 12.10 & 26.45 & $-2.02 *$ & 1.17 & -5.42 \\
\hline-6.04 & -3.91 & -6.86 & 11.17 & 16.25 & 11.75 & $6.29 * *$ & $8.72 * *$ & 0.00 \\
\hline-1.22 & 0.20 & 6.17 & 23.60 & 8.90 & 6.25 & -0.76 & 2.22 & -4.61 \\
\hline 1.70 & 3.47 & -7.14 & 11.19 & -4.64 & 2.01 & $-2.64 *$ & $-5.39 * *$ & -16.57 \\
\hline-0.41 & 0.00 & 9.50 & 17.29 & 1.69 & -7.42 & $2.93 *$ & 1.46 & 0.00 \\
\hline
\end{tabular}

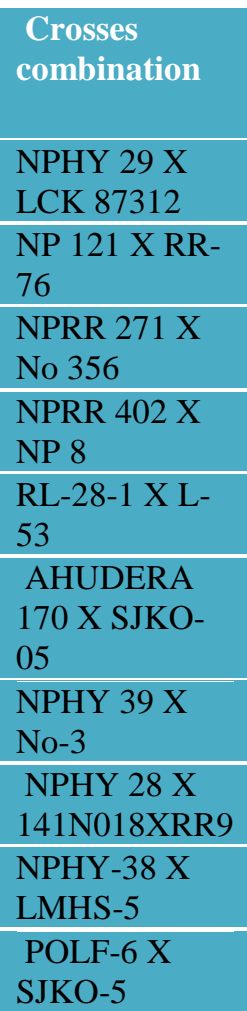

\begin{tabular}{|c|c|c|c|c|c|c|c|c|}
\hline \multicolumn{3}{|c|}{$\begin{array}{l}\text { Number of primary } \\
\text { branches per plant }\end{array}$} & \multicolumn{3}{|c|}{$\begin{array}{l}\text { Number of secondary } \\
\text { branches per plant }\end{array}$} & \multicolumn{3}{|c|}{$\begin{array}{c}\text { Number of capsules per } \\
\text { plant }\end{array}$} \\
\hline $\mathrm{BP}$ & MP & ID & BP & MP & ID & $\mathrm{BP}$ & MP & ID \\
\hline 0.00 & -17.24 & $\begin{array}{c}- \\
25.00\end{array}$ & 18.96 & 6.15 & 60.87 & 26.54 & 5.53 & 59.17 \\
\hline 44.44 & 13.04 & 15.38 & $113.15^{* *}$ & 39.65 & 55.55 & -18.72 & -23.32 & 64.05 \\
\hline-9.09 & -4.76 & 0.00 & 30.55 & -6.93 & 38.29 & $-37.40^{*}$ & $-31.30^{*}$ & 54.43 \\
\hline-27.27 & -40.74 & $\begin{array}{c}- \\
25.00\end{array}$ & $-46.42^{*}$ & $-41.74 *$ & 10.00 & -24.67 & -28.54 & 29.31 \\
\hline 50.00 & 5.88 & 22.22 & $57.57 * *$ & $28.39 *$ & 42.30 & $49.51 * *$ & $81.42 * *$ & 65.57 \\
\hline $110.00^{* * *}$ & 82.60 & 52.38 & $148.38 * *$ & $58.76^{* *}$ & 40.26 & $55.70 * *$ & $45.72 *$ & 67.15 \\
\hline $85.71 *$ & $116.66^{*}$ & 23.07 & $93.61 * *$ & $85.71 * *$ & 25.27 & $38.48 *$ & $35.80 *$ & 19.74 \\
\hline 58.33 & 52.00 & 21.05 & $125.00 * *$ & $120.00 * *$ & 59.59 & $46.35^{*}$ & $45.72 *$ & 47.50 \\
\hline $170.00 * *$ & $134.78 * *$ & 59.25 & $342.85^{* *}$ & $239.72 * *$ & 46.77 & $172.10 * *$ & $148.55^{* *}$ & 69.05 \\
\hline $92.30 * *$ & 47.05 & 20.00 & $77.77 * *$ & $49.53 * *$ & 25.00 & $109.89 * *$ & $90.54 * *$ & 33.49 \\
\hline
\end{tabular}


Table.1 Contd.

\begin{tabular}{|c|c|c|c|c|c|c|c|c|c|}
\hline \multirow{2}{*}{$\begin{array}{l}\text { Crosses } \\
\text { combinations }\end{array}$} & \multicolumn{3}{|c|}{ Number of seeds per capsule } & \multicolumn{3}{|c|}{ 1000- Seed weight (g) } & \multicolumn{3}{|c|}{ Seed yield per plant (g) } \\
\hline & BP & MP & ID & BP & MP & ID & $\mathrm{BP}$ & MP & ID \\
\hline $\begin{array}{l}\text { NPHY } 29 \mathrm{X} \\
\text { LCK } 87312\end{array}$ & -13.63 & -17.39 & -5.26 & 6.49 & 0.83 & 0.48 & $33.59^{* *}$ & $21.79^{*}$ & 35.39 \\
\hline $\begin{array}{l}\text { NP } 121 \text { X RR- } \\
76\end{array}$ & -5.00 & -5.00 & -10.52 & -1.51 & -6.32 & 0.82 & $43.41 * *$ & $50.42 * *$ & 41.83 \\
\hline $\begin{array}{l}\text { NPRR } 271 \mathrm{X} \\
\text { No } 356\end{array}$ & 0.00 & 0.00 & 20.83 & 6.49 & 3.94 & 9.78 & $46.14 * *$ & $37.16^{*}$ & 24.31 \\
\hline $\begin{array}{l}\text { NPRR } 402 \text { X } \\
\text { NP } 8\end{array}$ & -8.69 & -10.63 & 4.76 & -10.55 & -11.22 & -7.08 & 19.38 & 10.51 & 27.37 \\
\hline$\frac{\mathrm{RL}-28-1 \mathrm{X} \text { L- }}{53}$ & 4.76 & 2.32 & 22.72 & 5.88 & 3.17 & 2.11 & $50.57 * *$ & $18.95^{*}$ & 33.58 \\
\hline $\begin{array}{l}\text { AHUDERA } \\
170 \text { X SJKO-05 }\end{array}$ & 14.28 & 6.66 & 4.16 & -2.02 & -5.66 & -5.01 & $27.36^{* *}$ & $48.30 * *$ & 53.37 \\
\hline $\begin{array}{l}\text { NPHY } 39 \mathrm{X} \\
\text { No-3 }\end{array}$ & $\stackrel{-}{-}$ & $-18.36^{*}$ & -5.00 & -1.19 & -3.84 & 5.08 & $38.80^{* * *}$ & $18.29 *$ & 32.53 \\
\hline $\begin{array}{l}\text { NPHY } 28 \mathrm{X} \\
\text { 141N018XRR9 }\end{array}$ & 8.33 & 8.33 & 11.53 & 4.40 & -2.08 & -0.10 & $24.43^{*}$ & $24.09^{*}$ & 27.20 \\
\hline $\begin{array}{l}\text { NPHY-38 X } \\
\text { LMHS-5 }\end{array}$ & -11.53 & -6.12 & 13.04 & 4.08 & -5.66 & -0.48 & $30.40^{*}$ & $20.73^{*}$ & 14.78 \\
\hline $\begin{array}{l}\text { POLF-6 X } \\
\text { SJKO-5 }\end{array}$ & -8.69 & -12.50 & -4.76 & 2.37 & 3.53 & -74.84 & 9.07 & 7.77 & 23.25 \\
\hline \multirow{2}{*}{$\begin{array}{l}\text { Crosses } \\
\text { combinations }\end{array}$} & \multicolumn{3}{|c|}{ Oil content (\%) } & \multicolumn{3}{|c|}{ Protein content (\%) } & \multicolumn{3}{|c|}{ Palmitic acid } \\
\hline & BP & MP & ID & $\mathrm{BP}$ & MP & ID & $\mathrm{BP}$ & MP & ID \\
\hline $\begin{array}{l}\text { NPHY } 29 \mathrm{X} \\
\text { LCK } 87312\end{array}$ & 0.75 & -0.72 & 1.44 & 4.18 & -0.87 & -2.12 & $44.76 * *$ & $7.70^{*}$ & 36.42 \\
\hline $\begin{array}{l}\text { NP } 121 \text { X RR- } \\
76\end{array}$ & $12.91 * *$ & $13.59 * *$ & -9.10 & $10.33 * *$ & $8.50^{*}$ & 1.42 & $17.84 * *$ & $-4.61 *$ & 12.27 \\
\hline $\begin{array}{l}\text { NPRR } 271 \mathrm{X} \\
\text { No } 356\end{array}$ & $-6.48 * *$ & $-4.13 *$ & 1.31 & $8.44^{*}$ & $8.22 *$ & 3.29 & $97.10^{*}$ & $30.07 * *$ & 63.83 \\
\hline $\begin{array}{l}\text { NPRR } 402 \text { X } \\
\text { NP } 8\end{array}$ & $7.16^{* *}$ & 3.13 & 1.65 & 1.51 & -1.78 & 4.97 & $127.22 * *$ & $40.11 * *$ & 51.82 \\
\hline $\begin{array}{l}\text { RL-28-1 X L- } \\
53\end{array}$ & $\overline{-}^{-}$ & $\begin{array}{c}- \\
14.37 * *\end{array}$ & -8.90 & $19.25^{* *}$ & $20.69 * *$ & 11.63 & $42.13 * *$ & $18.20 * *$ & 38.51 \\
\hline $\begin{array}{l}\text { AHUDERA } \\
170 \text { X SJKO-05 }\end{array}$ & $-4.19^{*}$ & $-4.54 *$ & -3.77 & 4.80 & 1.48 & 4.96 & $84.24 * *$ & $17.34 * *$ & 16.15 \\
\hline $\begin{array}{l}\text { NPHY } 39 \mathrm{X} \\
\text { No-3 }\end{array}$ & $-8.49 * *$ & $-4.94 *$ & -3.15 & -3.62 & -4.88 & 1.64 & $-10.54^{*}$ & $-5.39 * *$ & -4.14 \\
\hline $\begin{array}{l}\text { NPHY } 28 \text { X } \\
\text { 141N018XRR9 }\end{array}$ & $-6.52 * *$ & $-7.89 * *$ & -5.58 & 4.51 & 3.96 & 0.92 & $90.99 * *$ & $18.30 * *$ & 37.72 \\
\hline $\begin{array}{l}\text { NPHY-38 X } \\
\text { LMHS-5 }\end{array}$ & 2.72 & -1.95 & -0.92 & -2.35 & 4.59 & -0.22 & -11.29 & $23.29 * *$ & -8.95 \\
\hline $\begin{array}{l}\text { POLF-6 X } \\
\text { SJKO-5 }\end{array}$ & $-7.87 * *$ & $-8.30 * *$ & -8.09 & 7.97 & 5.59 & 5.87 & 6.42 & 2.33 & -10.73 \\
\hline
\end{tabular}


Table.1 Contd.

\begin{tabular}{|c|c|c|c|c|c|c|c|c|c|}
\hline \multirow{2}{*}{$\begin{array}{l}\text { Crosses } \\
\text { combinations }\end{array}$} & \multicolumn{3}{|c|}{ Stearic acid } & \multicolumn{3}{|c|}{ Oleic acid } & \multicolumn{3}{|c|}{ Linoleic acid } \\
\hline & BP & MP & ID & BP & MP & ID & $\mathrm{BP}$ & MP & ID \\
\hline $\begin{array}{l}\text { NPHY } 29 \text { X LCK } \\
87312\end{array}$ & $8.66^{* *}$ & -1.05 & -4.18 & $83.73 * *$ & $36.19 * *$ & 55.23 & $-44.41 * *$ & $-30.65 * *$ & 249.37 \\
\hline NP 121 X RR-76 & $76.39 * *$ & $18.46^{* *}$ & 27.58 & $17.41 * *$ & $-20.84 * *$ & -83.57 & $13.72 *$ & 7.98 & 34.56 \\
\hline NPRR 271 X No 356 & $10.95 * *$ & $4.81 * *$ & -91.44 & $32.77 * *$ & $-31.33 * *$ & -72.71 & $158.70 * *$ & $80.08 * *$ & -18.28 \\
\hline NPRR $\angle$ & $63 . \overline{-}^{-}$ & $\begin{array}{c}- \\
46.60 * *\end{array}$ & 334.66 & $21.67 * *$ & $12.62 * *$ & 22.02 & 3.75 & -1.49 & 9.45 \\
\hline RL-2 & $-62.41 *$ & $-53.08 *$ & $\begin{array}{c}- \\
117.99\end{array}$ & $16.75^{* *}$ & 0.36 & -25.05 & -6.03 & -8.73 & -1.01 \\
\hline $\begin{array}{l}\text { AHUDERA } 170 \mathrm{X} \\
\text { SJKO-05 }\end{array}$ & $28.65^{* *}$ & $\begin{array}{c}- \\
38.66^{* *}\end{array}$ & -8.15 & $17.34 * *$ & $-35.56 * *$ & -124.84 & 5.35 & 3.97 & 45.54 \\
\hline NPHY 39 X No-3 & -0.35 & $-7.74 * *$ & 1.70 & $10.09 * *$ & $-8.45^{* *}$ & -20.92 & $-15.67 *$ & $-15.89^{*}$ & -12.96 \\
\hline $\begin{array}{l}\text { NPHY } 28 \mathrm{X} \\
\text { 141N018XRR9 }\end{array}$ & $82.64 * *$ & $53.87 * *$ & 18.93 & $44.60 * *$ & $-56.99 * *$ & -318.57 & $288.62 * *$ & $52.36 * *$ & 68.62 \\
\hline NPHY-38 X LMHS-5 & $65.93 * *$ & $13.34 * *$ & 39.95 & $7.12 * *$ & $40.96 * *$ & 44.04 & $27.34 * *$ & 9.60 & -66.62 \\
\hline POLF-6 X SJKO-5 & $44.62 * *$ & $\stackrel{-}{17.09 * *}$ & -42.77 & $8.64 * *$ & $3.52 * *$ & -11.67 & $-38.98 * *$ & $-32.26^{*}$ & -78.92 \\
\hline
\end{tabular}

\begin{tabular}{|l} 
Crosses combinations \\
\hline NPHY 29 X LCK 87312 \\
\hline NP 121 X RR-76 \\
\hline NPRR 271 X No 356 \\
\hline NPRR 402 X NP 8 \\
\hline RL-28-1 X L-53 \\
\hline AHUDERA 170 X SJKO-05 \\
\hline NPHY 39 X No-3 \\
\hline NPHY 28 X 141N018XRR9 \\
\hline NPHY-38 X LMHS-5 \\
\hline POLF-6 X SJKO-5 \\
\hline
\end{tabular}

\begin{tabular}{|c|c|c|}
\hline & Linolinic acid & \\
\hline BP & MP & ID \\
\hline 0.01 & -6.14 & 3.98 \\
\hline $9.51 * *$ & $3.95 * *$ & 5.38 \\
\hline $5.60 *$ & $-10.85 * *$ & 6.47 \\
\hline$-23.69 * *$ & $-13.68 * *$ & -28.03 \\
\hline $6.17 * *$ & $3.83 * *$ & 8.38 \\
\hline $32.12 * *$ & $12.18 * *$ & 15.36 \\
\hline $19.45 * *$ & $8.50 * *$ & 11.09 \\
\hline$-19.79 * *$ & $-11.22 * *$ & -15.16 \\
\hline$-1.28 *$ & $-16.10 * *$ & -8.87 \\
\hline $9.23 * *$ & $3.75 * *$ & 21.18 \\
\hline
\end{tabular}

All the crosses, NPHY 29 X LCK 87312, NP121 X RR 76, NPRR $271 X$ NO 356, NPRR $402 \quad X$ NP 8, RL-28-1 X L-53, AHUDEARA 170 X SJKO 05, NPHY 39 X NO 3, NPHY 28 X 141NO18XRR9, NPHY 38 LMHS-5 and POLF 10 X SJKO 55 showed significant increase.

Such observations were reported by VishnuAmeta et al., (2005); Singh et al., (2005); Chauhan et al., (2006); Tripathi et al.,(2011); Rao et al., (2011); Kumar et al., (2014) and Yadv et al., (2000).

\section{Oil content (\%)}

The range of heterosis over better parent was observed from -12.91 to 11.18 percent. Out of 10 crosses, only one cross NPRR 402 X NP 8 showed significant positive heterosis. The heterosis over mid parent varied from -14.37 to 3.13 per cent. Out of 10 crosses, none of them showed significant positive heterosis.

The range of inbreeding depression varied from -0.92 to -9.10 per cent. Seven crosses, NP121 X RR 76, RL-28-1 X L-53, 
AHUDEARA 170 X SJKO 05, NPHY 39 X NO 3, NPHY 28 X 141NO18XRR9, NPHY 38 LMHS-5 and POLF $10 \mathrm{X}$ SJKO 55 showed significant decrease while three combinations namely, NPHY 29 X LCK 87312, NPRR 271 X NO 356 and NPRR 402 $\mathrm{X}$ NP 8 showed significant increase.

\section{Protein content $(\%)$}

The range of heterosis over better parent observed from -2.35 to 19.25 per cent. Out of 10 crosses, only two crosses NPRR 271 X NO 356 and RL-28-1 X L- 53 showed singificant positive heterosis.

The heterosis over mid parent varied from 0.87 to 20.69 per cent. Out of 10 crrosses, NPRR271 X NO 356 and RL-28-1 X L-53 showed significant positive heterosis.

The range of inbreeding depression varied from -0.22 to 11.63 per cent. Two crosses NPHY 29 X LCK 87312 and NPHY 38 LMHS-5 showed significant decrease while eight combinations namely, NP121 X RR 76, NPRR 271 X NO 356, NPRR 402 X NP 8, RL-28-1 X L-53, AHUDEARA 170 X SJKO 05, NPHY $39 \quad X \quad N O \quad 3$, NPHY $28 \quad X$ 141NO18XRR9 and POLF $10 \mathrm{X}$ SJKO 55 showed significant increase.

\section{Palmitic acid}

The range of heterosis over better parent observed from 6.42 to 90.99 per cent. Out of 10 crosses, six crosses NPHY 29 X LCK 87312, NP121 X RR 76, NPRR271 X NO 356, RL-28-1 X L-53, AHUDER $170 \mathrm{X}$ SJKO 05 and NPHY 28 X 141NO18XRR9 showed significant positive heterosis.

The heterosis over mid parent varied from 4.61 to 40.11 per cent. Out of 10 crosses, NPRR271 X NO 356, NPRR 402 X NP 8, RL-28-1 X L-53, AHUDER 170 X SJKO 05 and NPHY 28 X 141NO18XRR9 showed significant positive heterosis.

The range of inbreeding depression varied from -8.95 to 63.83 per cent. Three crosses NPHY 39 X NO 3, NPHY 38 LMHS-5 and POLF 10 X SJKO 55 showed significant decrease while seven combinations namely, NPHY 29 X LCK 87312, NP121 X RR 76, NPRR $271 X$ NO 356, NPRR 402 X NP 8, RL-28-1 X L-53, AHUDEARA 170 X SJKO 05 and NPHY 28 X 141NO18XRR9 showed significant increase.

\section{Stearic acid}

The range of heterosis over better parent was observed from -0.35 to 82.64 per cent. Out of 10 crosses, six crosses NP121 X RR 76, NPRR $271 X$ NO 356, AHUDER $170 X$ SJKO 05, NPHY 28 X 141NO18XRR9, NPHY 38 X LMHS-5 and POLF $10 X$ SJKO 55 showed significant positive heterosis for stearic acid.

The heterosis over mid parent varied from 1.05 to 53.87 per cent. Out of 10 crosses, NP 121 X RR 76, NPRR271X NO 356, NPHY 28 X 141NO18XRR9 and NPHY 38 X LMHS-5 showed significant positive heterosis.

The range of inbreeding depression varied from 1.70 to 39.95 per cent. Six crosses, NPHY 29 X LCK 87312, NPRR 271 X NO 356, NPRR 402 X NP 8, RL-28-1 X L-53, AHUDEARA 170 X SJKO 05 and POLF 10 $X$ SJKO 55 showed significant decrease while four combinations namely, NP121 X RR 76, NPHY $39 \quad \mathrm{X} \quad \mathrm{NO} \quad 3$, NPHY $28 \quad \mathrm{X}$ 141NO18XRR9 and NPHY 38 LMHS-5 showed significant increase.

\section{Oleic acid}

The range of heterosis over better parent was observed from 7.12 to 82.73 per cent. Out of 
10 crosses, nine crosses NPHY 29 X LCK 87312, NP121 X RR 76, NPRR 402 X NP 8, RL-28-1 X L-53, AHUDER 170 X SJKO 05, NPHY $39 \quad X \quad$ NO 3 , NPHY $28 \quad X$ 141NO18XRR9 and POLF 10 X SJKO 55 showed significant positive heterosis.

The heterosis over mid parent varied from 0.36 to 40.96 per cent. Out of 10 crosses, NPHY 29 X LCK 87312, NPRR 402 X NP 8, NPHY $38 X$ LMHS-5 and POLF 10 X SJKO 55 showed significant positive heterosis.

The range of inbreeding depression varied from -11.67 to 55.23 per cent. Seven crosses, NP 121 X RR 76, NPRR 271 X NO 356, RL28-1 X L-53, AHUDEARA 170 X SJKO 05, NPHY $39 \quad X \quad$ NO 3 , NPHY $28 \quad X$ 141NO18XRR9 and POLF 10 X SJKO 55 showed significant decrease while three combinations namely, NPHY 29 X LCK 87312, NPRR 402 X NP 8 and NPHY 38 X LMHS-5 showed significant increase.

\section{Linoleic acid}

The range of heterosis over better parent was observed from 3.75 to 288.62 per cent. Out of 10 crosses, four crosses NP121 X RR 76, NPRR $271 \quad X \quad N O \quad 356$, NPHY $28 \quad X$ 141NO18XRR9 and NPHY 38 X LMHS-5 55 showed significant positive heterosis.

The heterosis over mid parent varied from 1.49 to 80.08 per cent. Out of 10 crosses, NPRR 271 X NO 356 and NPHY 28 X 141NO18XRR9 showed significant positive heterosis.

The range of inbreeding depression varied from -1.01 to 34.56 per cent. Six crosses NPHY 29 X LCK 87312, NPRR 271 X NO 356, RL-28-1 X L-53, NPHY 39 X NO 3, NPHY 38 LMHS-5 and POLF 10 X SJKO 55 showed significant decrease while four combinations namely, NP 121 X RR 76,
NPRR 402 X NP 8, AHUDEARA $170 \mathrm{X}$ SJKO 05 and NPHY 28 X 141NO18XRR9 showed significant increase.

\section{Linolenic acid}

The range of heterosis over better parent was observed from 0.01 to 32.12 per cent. Out of 10 crosses, six crosses NP121 X RR 76, NPRR $271 X$ NO 356, RL-28-1 X L-53, AHUDER 170 X SJKO 05, NPHY 39 X NO 3 and POLF $10 \mathrm{X}$ SJKO 55 showed significant positive heterosis.

The heterosis over mid parent varied from 3.83 to 12.18 per cent. Out of 10 crosses, NP121 X RR 76, RL-28-1 X L-53, AHUDER $170 \mathrm{X}$ SJKO 05, NPHY $39 \mathrm{X}$ NO 3 and POLF 10 X SJKO 55 showed significant positive heterosis.

The range of inbreeding depression varied from 3.98 to 21.18 per cent. Three crosses, NPRR 402 X NP 8, 28 X 141NO18XRR9 and 38 LMHS-5 showed significant decrease while seven combinations namely, NPHY 29 X LCK 87312, NP121 X RR 76, NPRR 271 $X$ NO 356, RL-28-1 X L-53, AHUDEARA 170 X SJKO 05, NPHY 39 X NO 3 showed significant increase.

\section{References}

Anonymous, 1975. Methods of analysis, $12^{\text {th }}$ edition, Association of official analytical chemists, Washington, D.C.

Anonymous, 2014-15. Food and Agriculture organization of the United Nation. FAO Statistics. (www.faostat.org.com).

Chauhan, V.S.; Kumar, M.P. and Singh, R. B 2006. Heterosis for yield and yield attributes in linseed (Linum usitatissimum L.) Farm Science Journal, 15(1):29-31.

Kumar, S; Kumar, R; Kumar, S; Singh, S; Kumar, S and Singh, M. P. 2014. 
Estimation of heterosis in linseed (Linum usitatissimum L.). International Journal of Agricultural Sciences, 10(1):356-359.

Luddy E F, Barforad R A, Berbs S F \& Magidman P 1968. J Amer Oil Chem Soc, 65, 549.

Patil, V. D and Chopde, P. R. 1983. Heterosis in relation to GCA and SCA effects in linseed. Indian J. Genet, 43 (2): 226228.

Rao, D. G and Pandagare. S. S. 2011. Genetic analysis of yield and yield attributing characters in linseed (Linum usitatissimum L.) Asian Journal of Bio Science, 6(1): 16-22.

Rao, S. S.; Rede, A. P and Chandrakar, P. K. 2001. Heterosis and inbreeding depression in linseed. (Linum usitatissimum L.). Journal of Agricultural Sciences, 35(1):16-19.

Saraswat, A.V and Kumar, S. 1993. Heterosis and inbreeding depression in some early hybrids of linseed. In heterosis breeding in crop plant-theory and application: short communications: symposium Ludhiana 23-24 pp.

Shull, G.H. 1908. The composition of a field of maize. Rept. Amer. Breeder's Assoc. 4: 296-301.

Singh, P; Singh, D and Singh, S. K 2005. Heterosis in relation to other genetic parameters in linseed. Farm-ScienceJournal, 14(2): 1-3.

Tripathi, S; Mishra, V and Tripathi, H. C. 2011. Heterosis and inbreeding depression in relation to other genetic parameters for yield and its attributes in Linseed (Linum usitatissimum L.). New Agriculturist, 22(2):207-210.

Verma, A. K and Sinha, P. K. 1993. Heterosis in linseed. In heterosis breeding in crop plants. Theory and application: short communication: symposium Ludhiana, 23-24 Feb. 1993.

Vishnu, A; Shah, M. A and Lakshyadeep. 2005. Studies on heterosis and combining ability for fatty acids in linseed (Linum usitatissimum L). Electronic journal of Plant Breeding, 5(1):127-129.

Yadav, R. K 2000. Heterosis for yield and yield components in linseed (Linum usitatissimum L.). Plant Archives 1(1\&2): 95-98.

\section{How to cite this article:}

Yadav, P.C., R.K. Yadav, Vishwanath, Yogesh Pandey and Sanjeev Kumar. 2018. Heterosis and Inbreeding Depression for Seed Yield and Its Related Morphological Traits in Linseed (Linum usitatisimum L.). Int.J.Curr.Microbiol.App.Sci. 7(01): 3088-3098. doi: https://doi.org/10.20546/ijcmas.2018.701.366 